\title{
Correction for: Suppressing the KIF20A/NUAK1/Nrf2/GPX4 signaling pathway induces ferroptosis and enhances the sensitivity of colorectal cancer to oxaliplatin
}

\author{
Changshun Yang ${ }^{1, *}$, Yu Zhang ${ }^{2,}{ }^{*}$, Shengtao Lin ${ }^{1, *}$, Yi Liu ${ }^{3}$, Weihua Li $^{1}$ \\ ${ }^{1}$ Department of Surgical Oncology, Fujian Provincial Hospital, Fuzhou 350001, China \\ ${ }^{2}$ Department of Pathology, The First Affiliated Hospital of Fujian Medical University, Fuzhou 350001, China \\ ${ }^{3}$ Department of Endoscopy, National Cancer Center/Cancer Hospital, Chinese Academy of Medical Science and \\ Peking Union Medical College, Beijing 100000, China \\ *Equal contribution
}

Correspondence to: Weihua Li; email: liwh68@sina.com

Original article: Aging (Albany NY) 2020; 13: 13515 - 13534

PMID: $33819186 \quad$ PMCID: PMC7950286 DOI: 10.18632/aging.202774

This article has been corrected: The authors requested to update Funding information. The authors declare that these corrections do not change the results or conclusions of this work.

\section{FUNDING}

This work was supported by the Youth Scientific Research Project of Fujian Provincial Health, Family Planning Commission (grant number 2018-2-5) and the Sail Fund of Fujian Medical University (grant number 2017XQ1151), and Foundation of 2020 Fujian Provincial Department of Finance Health and Health Provincial Special Subsidy. 